\title{
A Detailed Checklist in Cardio-Thoracic Surgery: The Isala safety Check
}

\author{
Sander Spanjersberg ${ }^{1 *}$, Jan Paul Ottervanger ${ }^{2}$, Arno Nierich ${ }^{1}$, Marieke de Boer ${ }^{3}$ and George Brandon Bravo \\ Bruinsma $^{4}$ \\ ${ }^{1}$ Department of Anesthesiology and Intensive Care, Isala, Zwolle, Europe \\ ${ }^{2}$ Department of Cardiology, Isala Heart Centre, Europe \\ ${ }^{3}$ Department of Quality and Safety, Isala, Zwolle, Europe \\ ${ }^{4}$ Department of Cardiothoracic Surgery, Isala Heart Centre, Europe
}

Submission: November 21, 2016; Published: December 01, 2016

*Corresponding author: Sander Spanjersberg, intensivist/cardiothoracic-anesthesiologist, ISALA Hospital, ISALA Heart Centre, Dokter van Heesweg 2, Postbus 105000, 8000 GM Zwolle, The Netherlands, Europe, Tel: + 3138 4244216; Email: a.j.spanjersberg@isala.nl

\begin{abstract}
In various fields of complex environments, checklists have been introduced, mainly to improve procedural related aspects like logistics, personnel support and equipment. However, in order to pursue better outcome, major patient-related factors may be likewise important to check immediately before surgical procedures. Therefore, a specific checklist, the Isala Safety Check, was developed in a high-volume cardiothoracic surgery unit. It concerns a short list focusing on the presence of potential sources for peri-operative complications, including actual information of cardiac function, pulmonary comorbidities, renal impairment, neurological condition, predisposing factors for postoperative infections and risk of transfusion. Special attention is paid to the on-site information obtained by standard transesophageal echo, particularly the visualization of the condition of the ascending aorta. After anesthesia induction, but just before skin incision, these items are discussed in the presence of the entire team, by means of a checklist. Consensus is achieved to whether there are any adaptations required for the operation. This method has shown to increase team awareness, stimulate communication and may improve outcome. Details of the Isala Safety Check and the application of this checklist in routine and emergency cardiac surgery are discussed.
\end{abstract}

Keywords: Cardiac surgery; checklist; time out; patient safety; risk factors; team approach

Abbreviations: TEE: Transesophageal Echo; WHO: World Health Organization; GFR: Glomerular Filtration Rate

\section{Introduction}

Careful pre-procedural evaluation of patients is one of the cornerstones of preventing complications in many complex procedures, including cardio-thoracic surgery. A stop moment, like the time-out immediately before surgery, has demonstrated its importance and has become routine in many complex teamdriven specialties, in medical and non-medical surroundings [1]. In addition, several specific checklists have been developed, and proved highly effective in decreasing complications [2]. However, for cardio-thoracic surgery patients important items are not addressed during a standardized time-out. Also, time-out checklists focus primarily on procedural issues and still lack optimal communication about patient specific risk factors between the team members before incision [2]. This of interest, because the current guidelines provide only a few recommendations of evaluating patients for cardio thoracic surgery [3]. Although our unit has established good outcome results over many years, we developed an extended checklist. This is based on the fact that a substantial number of cardiac surgery related deaths is avoidable [4]. Introducing a specific checklist, by professionals and intended for professionals, covering patient-specific items with respect to risk factors for perioperative complications, we assumed to improve patientrelated outcome and team performance [5]. The checked items are discussed just before skin incision, because at this moment all information is up-to-date and complete, including standard pre-procedural transesophageal echo (TEE), and there is still an opportunity to adapt the surgical strategy. Details of the Isala Safety Check, backgrounds and suggestions for implementation are discussed in this paper. 


\section{Standard evaluation of cardiothoracic patients}

The routine pre-operative assessment of cardiac patients for non-cardiac surgery is well studied and described [6]. However, there is limited literature on the content of a structured routine pre-operative evaluation of patients for cardiothoracic surgery. Furthermore, although the guidelines give only few recommendations, particularly on pulmonary and renal function, a structured approach to a complete pre-operative evaluation is lacking and therefore may be variable [3].

\section{Time-out procedure}

Table 1: Time-out procedure.

\begin{tabular}{|c|c|}
\hline Time-out & patient identification \\
\hline & correct operation \\
\hline & correct site and side \\
\hline & allergies \\
\hline & comorbidity (not specified) \\
\hline & hemostatic status \\
\hline & antibiotic profylaxis needed and \\
& given \\
\hline & materials, equipment, personnel \\
\hline & patient positioning \\
\hline
\end{tabular}

Signed by surgeon, in presence of patient, anesthesia assistant, surgeon, anesthesiologist, or assistant

The World Health Organization (WHO) has developed a surgical safety checklist in 2005. This checklist has found wide adoption and is now used in all fields of surgery even in minimal invasive procedures [7]. Since 2009, a standard timeout procedure is mandatory in all Dutch hospitals for every surgical intervention [8]. The checklist for this procedure is summarized in (Table 1). This checklist focuses on procedural

\section{Contents of the Isala Safety check}

Table 2: Isala Safety Check.

\begin{tabular}{|c|c|c|c|c|}
\hline step 1 & & & & \\
\hline & $\begin{array}{l}\text { time-out procedure } \\
\text { followed }\end{array}$ & & yes & no \\
\hline \multicolumn{5}{|l|}{ step 2} \\
\hline & Euro score (standard) & low & intermediate & high \\
\hline \multirow[t]{7}{*}{ step 3} & risk factors & no & yes & discuss \\
\hline & cardiac & & & \\
\hline & pulmonary & & & \\
\hline & renal & & & \\
\hline & neurologic & & & \\
\hline & infectious & & & \\
\hline & hemostatic & & & \\
\hline \multirow[t]{2}{*}{ step 4} & Safety items TEE & & & \\
\hline & LV function/LAA/OFO & & & \\
\hline
\end{tabular}

aspects. Checking these items minimizes wrong patient/ procedure/site complications and prevents intra-operative delay due to equipment problems. The time-out procedure has proven its effectiveness in the field of mixed surgical population, general surgery, orthopedic surgery, otorhinolaryngology and neurosurgery [9-11]. Despite a few negative reports [12], a significant reduction was demonstrated in overall complication rate and even in mortality [13]. In the SURPASS study, the WHO checklist was extended to a multistage, multidiscipline document covering the entire patient course from admission to discharge. This approach reduced in hospital mortality from 1.5 to $0.8 \%$ and total complication rate from 27.3 to $16.7 \%$ [2].

\section{Limitations of the time-out}

Although the WHO surgical checklist reduced both complication rate and mortality, there are some limitations of this approach. Whereas it focuses on the procedural aspects, communication on patient information concerning risk factors and comorbidity might receive little attention. Furthermore, a checklist on itself does not require interaction or discussion [14]. Therefore, there is a possibility that after the first introduction of a checklist the behavior of the performing team changes into a "ticking" culture instead of actual awareness and concern for the situation [15]. In Ontario, the introduction of the surgical safety checklist has not led to a measurable change in outcome [12]. One of the reasons might have been that, besides the short period of evaluation, the standard checklist is not directly calling for actions by all members of the operating team [16]. Finally, the surgical safety checklist is, by design, a general operation or patient category are lacking. Therefore, checklists especially designed for certain operations, like transphenoidal neurosurgery, were developed [17-18]. Cardiac surgery is also such a type of operation, with specific risk factors and a patient population with extensive comorbidity, requiring a specific cardiac surgery checklist. checklist. This implies that items concerning a specific type of 


\begin{tabular}{|l|c|l|l|l|}
\hline & Valves & & \\
\hline & Ascending aorta & & & \\
\hline & adapt procedure & no & yes & discuss \\
\hline & IABP & & & \\
\hline & step 5 & & & \\
\hline & canulation strategy & & & \\
\hline & ECC adaptation & & & \\
\hline & proximal anastomosis & & & \\
\hline & intra cardiac adaptation & & & \\
\hline & extra cardiac adaptation & & & \\
\hline & $\begin{array}{c}\text { no surgery, stop } \\
\text { procedure }\end{array}$ & & & \\
\hline & other & & & \\
\hline
\end{tabular}

The items that are checked are represented in (Table 2). It is essential that the total procedure is done by the complete team, comprising the cardiac surgeon, the assisting, scrub nurse, anesthesiologist, anesthetic nurse and perfusionist. The first checked item by the team is whether the regular time-out was indeed performed. If the regular time-out procedure was not performed, the surgeon is urged to perform. Especially in the setting of life threatening emergency cases this step might have been skipped initially but should be performed at this moment, or it should be documented that the team decides that the regular time-out cannot be performed. To increase the awareness for every team member with regard to the expected mortality of the procedure, the calculated EuroSCORE is repeated. Accordingly, patients are divided into three groups: low, intermediate and high risk [19]. The third step is to identify other specific peri- and postoperative risks and complications with focus on six main organ specific topics: cardiac, pulmonary, renal, neurologic, inflammation and coagulation. Cardiac risk factors include reduced ventricular function, hypertrophy, critical coronary artery stenosis, intra-cardiac shunts, valvular dysfunction and pulmonary hypertension. Each of these findings may require additional measures.

Pulmonary risk factors include mainly COPD, bronchiectasis and restrictive pulmonary function. These conditions may urge to reduce tidal volume, focusing on lung protective ventilation and careful fluid balance [20]. Increased risk for renal dysfunction may arise from an already decreased glomerular filtration rate (GFR), but also previous periods of (reversible) renal failure. Anticipation may involve the use of additional filtration on the extracorporeal circuit or targeted perfusion pressure [21]. It may be indicated that postoperative strategies are discussed here already. Factors that lead to a higher risk of adverse neurologic outcome are discussed. Previous stroke, transient ischemic attacks and known carotid artery stenosis are the most prominent factors. Preventive measures may include higher perfusion pressures, additional monitoring of the ascending aorta and postoperative anticoagulant therapy. Conditions that increased the risk of infectious complications are discussed, like diabetes, depressed immune system, urinary tract problems or chronic pulmonary disease. Special attention is paid to the risk factors for postoperative wound infection. Preventive measures include avoidance of bilateral mammalian artery use and continued use of antibiotics postoperatively. Cardiac surgery interferes with the hemostatic system and patients are mostly on anti-coagulant therapy. Careful discussion on the management of this therapy is mandatory. Therefore, the risk factors for hemostatic complications and possible preventive measures are to be discussed.

The fourth step of the Isala Safety check consists of information from actual transesophageal echocardiography (TEE) images, obtained immediately after induction of anesthesia. The required images include a stepwise identification of global myocardial contractility, possible sources of intra-cardiac emboli, the oval foramen, all cardiac valves and the ascending aorta. If any atherosclerosis with a grade of 3 or more based on the Katz classification [22] of the visible part of the ascending aorta or descending aorta is found, additional imaging of the ascending aorta using modified TEE [23] should be considered in order to increase the diagnostic accuracy of TEE [24]. The results of this so-called focused TEE investigation are discussed within the team. The fifth and final step is to summarize and discuss the findings of the first steps, and decide whether an adaptation of the surgical plan is necessary. This can vary from additional monitoring by epi-aortic scanning in order to guide changes in the canulation or clamping site, or to a total change in surgical approach, as example change from on-pump CABG to off-pump CABG.

\section{How to use the Isala Safety check}

The Isala Safety check is performed after induction of anesthesia, just prior to skin incision. This moment is chosen because at this time all information is available, whereas there is still a possibility to change the strategy for the operation and even to stop the operation. It is essential that all members of the team are present, including perfusionist, scrub nurse, surgical assistant, anesthetic nurse, surgeon and anesthesiologist. The 
anesthesiologist is in the lead together with the surgeon in communicating about the safety check items. Since all activities are paused during the checklist maximum awareness is ensured. Most patients have elective surgery, and in these patients the Isala Safety check can be performed, with minimal time loss. A YouTube demonstration has shown that the team communication will take only $2.09 \mathrm{~min}$ and no additional operative time is needed to obtain the specific TEE images. Although the Isala Safety Check is primarily designed for elective surgery, emergent cases may benefit even more, particularly because most complications occur in emergent cases. Monitoring the results of the Isala Safety check, by registering adaptations to the operation plan in combination with outcome of surgery, creates a feedback loop. Regular update of this information to the healthcare providers may lead to better preparation of patients and a tapered workup for specific groups of patients.

\section{Limitations Safety Check}

The first potential limitation is time loss, due to both numerating the comorbidity of the patient and the assessment of a dedicated TEE examination. However, all items addressed in the Isala Safety Checklist are notified information in the patient file and the TEE is a focused examination that is performed during the surgical preparation phase with no increase of operative time. The second limitation is that the Safety Check is performed just before skin incision, being late in the entire work-up process. Changing the operation procedure or even stop the procedure at this moment will have major (emotional, logistic and financial) effects. However, this is the final moment to prevent major complications and a sub-optimal approach. The fact that TEE is performed during general anesthesia, may influence myocardial and valve function, and interpretation should be done with caution. However, experienced echo cardiographers are aware of this limitation. A counter effect of checking important items and offering the opportunity to adapt the surgical plan is that ad hoc decisions may be taken. Although this risk is present, the Isala Safety Check is not performed to replace the heart team, where cardiologist and cardiothoracic surgeon decide together the best treatment strategy for this patient. However, in the case of unknown or changed important findings, it may be unethical to ignore this.

If patients are not informed about the performance of the Isala Safety Check and the possible consequences and adaptations of the operation plan, this may interfere with the consent of the patient. Therefore, it is mandatory to inform the patients of this checklist and the possible consequences, and to verify that patients agree with its use. Other items may be added to the content of the Isala Safety Checklist in the future when the outcome of the data show that certain adaptations appear more in specific patient groups. It may be mandatory for these selected groups to adjust the work up for certain operations in order to realize a safer surgical strategy. Furthermore, the outcome of the check is depending on the quality and the interpretation of the echo findings and the culture in the operating room to discuss the strategy. This necessitates skilled professionals and an open, respectful atmosphere where everyone's contribution is appreciated. A final limitation is that until now the benefit of the Isala Safety Check has not been demonstrated in a randomized controlled trial. However, the concept of providing a safer cardiac operation by applying the check will rise no doubt at all.

\section{Future recommendations}

With reference to the results of checklists in various fields where professionals perform high-tech interventions in multi disciplinary teams, it is our believe that the Isala Safety Check in combination with monitoring results, discussing complications, a learning attitude and focus on teamwork will reduce both mortality and complication rate. Evidence based medicine is the standard of care, and therefore we advocate a registry of the implementation of the Isala Safety Check, including the adaptations to the original operation plan. Many checklists have been introduced and evaluated. To our knowledge, a randomized clinical trial has not been published; probably due to fact that double blinding is impossible.

\section{Conclusion}

Surgical checklists have proven to reduce perioperative complications. The addition on top of the timeout procedure of a specific checklist for cardio-thoracic surgery may increase the awareness of the team of potential pitfalls, and enable change of the surgical approach. The Isala Safety Checklist offers an opportunity to systematically address specific risk factors and discuss possible interventions. Timing of the checklist just before skin incision adds actual cardiac information derived from the images of the pre-operative transesophageal echo to the data of the patient's history, and creates the opportunity to adapt the surgical plan to a safe strategy. Future research is necessary to evaluate the added value of the Isala Safety Check in the outcome of cardio thoracic surgery patients.

\section{References}

1. Gawande A (2016) The Checklist Manifesto-How to Get Things Right. Metropolitan Books.

2. Eefje N de Vries, Hubert A Prins, Rogier MPH Crolla, Adriaan J den Outer, George van Andel, et al. (2010) Effect of a comprehensive surgical safety system on patient outcomes. New England Journal of Medicine 363(20): 1928-1937.

3. (2014) ESC/EACTS Guidelines on myocardial revascularization. Eur Heart J 35(37): 2541-2619.

4. Gawande AA, Thomas EJ, Zinner MJ, Brennan TA (1999) The incidence and nature of surgical adverse events in Colorado and Utah in 1992. Surgery 126(1): 66-75.

5. Russ S, Rout S, Sevdalis N, Moorthy K, Darzi A, et al. (2013) Do safety checklists improve teamwork and communication in the operating room? A systematic review. Ann Surgery 258(6): 856-871.

6. (2014) ESC/ESA Guidelines on non-cardiac surgery: cardiovascular assessment and management. Eur Heart J 35(35): 2383-2431. 
7. Patel J, Ahmed K, Guru KA, Khan F, Marsh H, et al. (2014) An overview of the use and implementation of checklists in surgical specialities - a systematic review. Int J Surg 12(12): 1317-1323.

8. Inspectorate DH (2008) Standardization is crucial to reduce operative risks [Dutch]. P. 1-80.

9. Haugen AS, Søfteland E, Almeland SK, Sevdalis N, Vonen B, et al. (2015) Effect of the World Health Organization Checklist on Patient Outcomes. Ann Surgery 261(5): 821-828.

10. Enchev Y (2015) Checklists in Neurosurgery to Decrease Preventable Medical Errors: A Review. Balkan Med J 32(4): 337-346.

11. Russ S, Rout S, Caris J, Mansell J, Davies R, et al. (2015) Measuring variation in use of the WHO surgical safety checklist in the operating room: a multicenter prospective cross-sectional study. J Am Coll Surg 220(1): 1-11.

12. David R Urbach, Anand Govindarajan, Refik Saskin, Andrew S Wilton, Nancy N Baxter (2014) Introduction of Surgical Safety Checklists in Ontario, Canada. New England Journal of Medicine 370(11): 10291038.

13. van Klei WA, Hoff RG, van Aarnhem EE, Simmermacher RK, Regli LP et al. (2012) Effects of the Introduction of the WHO "Surgical Safety Checklist" on In-Hospital Mortality. Ann of Surgery 255(1): 44-49.

14. Wahr JA, Prager RL, Abernathy JH, Martinez EA, Salas E, et al. (2013) Patient Safety in the Cardiac Operating Room: Human Factors and Teamwork: A Scientific Statement From the American Heart Association. Circulation 128(10): 1139-1169.

15. Gillespie BM, Withers TK, Lavin J, Gardiner T, Marshall AP (2016) Factors that drive team participation in surgical safety checks: a prospective study. Patient Safety in Surgery 10(3): 1-9.

16. Leape LL (2014) The Checklist Conundrum. N Engl J Med 370(11): 1063-1064.
17. Christian E, Harris B, Wrobel B, Zada G (2014) Endoscopic endonasal transsphenoidal surgery: implementation of an operative and perioperative checklist. Neurosurgical Focus 37(4): E1.

18. Laws ER, Wong JM, Smith TR, de Los Reyes K, Aglio LS, et al. (2015) A checklist for endonasal transsphenoidal anterior skull base surgery. J Neurosurg 124(6): 1634-1639.

19. Roques F, Nashef SA, Michel P, Gauducheau E, de Vincentiis C, et al. (1999) Risk factors and outcome in European cardiac surgery: analysis of the EuroSCORE multinational database of 19030 patients. Eur J Cardiothorac Surg 15(6): 816-823.

20. Marseu K, Slinger P (2015) Peri-operative pulmonary dysfunction and protection. Anaesthesia 71(1): 46-50.

21. Hori D, Hogue C, Adachi H, Max L, Price J, et al. (2016) Perioperative optimal blood pressure as determined by ultrasound tagged near infrared spectroscopy and its association with postoperative acute kidney injury in cardiac surgery patients. Interact CardioVasc Thorac Surg 22(4): 445-451.

22. Katz ES, Tunick PA, Rusinek H, Ribakove G, Spencer FC, et al. (1992) Protruding aortic atheromas predict stroke in elderly patients undergoing cardiopulmonary bypass: experience with intraoperative transesophageal echocardiography. J Am Coll Cardiol 20(1): 70-77.

23. B van Zaane, AP Nierich, WF Buhre, GJ Brandon Bravo Bruinsma, KGM Moons (2007) Resolving the blind spot of transoesophageal echocardiography: a new diagnostic device for visualizing the ascending aorta in cardiac surgery. British Journal of Anaesthesia 98(4): 434-441.

24. Jansen Klomp WW, Peelen LM, Spanjersberg SJ, Brandon Bravo Bruinsma GJ, Lange Fd, et al. (2014) Added value of modified transoesophageal echocardiography in the diagnosis of atherosclerosis of the distal ascending aorta in cardiac surgery patients. Eur Heart J Cardiovasc Imaging 15(6): 623-630.

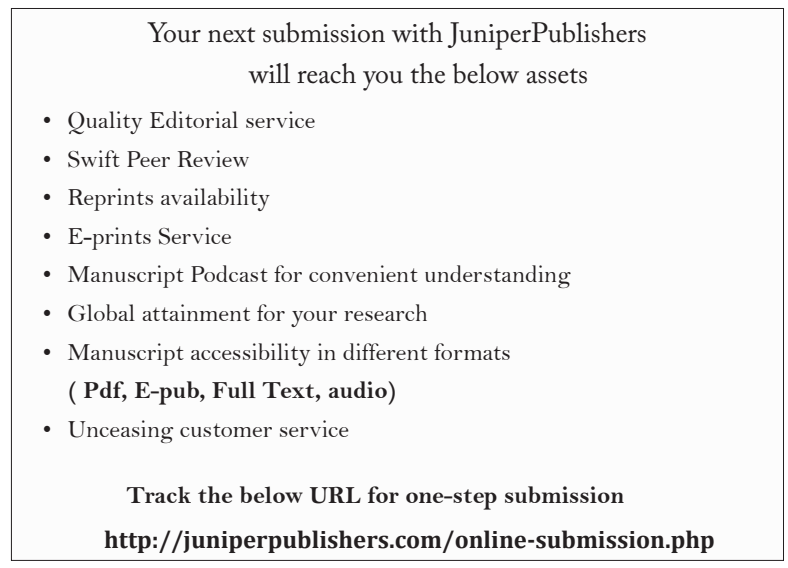

